\title{
Everolimus as first line therapy for pancreatic neuroendocrine tumours: current knowledge and future perspectives
}

\author{
Marco Gallo $^{1}\left(\mathbb{0} \cdot\right.$ Pasqualino Malandrino $^{2} \cdot$ Giuseppe Fanciulli $^{3} \cdot$ Francesca Rota $^{4}$. \\ Antongiulio Faggiano $^{5} \cdot$ Annamaria Colao $^{6} \cdot$ On behalf of NIKE Group
}

Received: 23 March 2017 / Accepted: 24 March 2017

(C) Springer-Verlag Berlin Heidelberg 2017

\begin{abstract}
Purpose Everolimus has been shown to be effective for advanced pancreatic neuroendocrine tumours (pNETs), but its positioning in the therapeutic algorithm for pNETs is matter of debate.

Methods With the aim to shed light on this point, we performed an up-to-date critical review taking into account the results of both retrospective and prospective published studies, and the recommendations of international guidelines. In addition, we performed an extensive search on the Clinical Trial Registries databases worldwide, to gather information on the ongoing clinical trials related to this specific topic.
\end{abstract}

Members of the NIKE Group is given in the Acknowledgement section.

Marco Gallo

mgallo4@cittadellasalute.to.it

1 Oncological Endocrinology Unit, Department of Medical Sciences, University of Turin, AOU Città della Salute e della Scienza di Torino, Via Genova 3, 10126 Turin, Italy

2 Endocrinology Unit, Garibaldi Nesima Medical Center, Catania, Italy

3 Neuroendocrine Tumours Unit, Department of Clinical and Experimental Medicine, University of Sassari, AOU Sassari, Sassari, Italy

4 Endocrinology Unit, Regina Elena National Cancer Institute, Rome, Italy

5 Thyroid and Parathyroid Surgery Unit, Istituto Nazionale per lo Studio e la Cura dei Tumori "Fondazione G. Pascale", IRCCS, Naples, Italy

6 Department of Clinical Medicine and Surgery, University "Federico II", Naples, Italy
Results We identified eight retrospective published studies, two prospective published studies, and five registered clinical trials. Moreover, we analyzed the content of four widespread international guidelines.

Conclusions Our critical review confirms the lack of highquality data to recommend everolimus as the first line therapy for pNETs. The ongoing clinical trials reported in this review will hopefully help clinicians, in the near future, to better evaluate the role of everolimus as the first line therapy for pNETs. However, at the moment, there is already enough evidence to recommend everolimus as the first line therapy for patients with symptomatic malignant unresectable insulin-secreting pNETs, to control the endocrine syndrome regardless of tumour growth.

Keywords Everolimus · Neuroendocrine tumours · mTOR inhibitors · Therapy

\section{Introduction}

Pancreatic neuroendocrine tumours (pNETs) are rare tumours with an incidence rate that is steadily increasing in different countries (Capelli et al. 2012; Scherubl et al. 2013; Tsai et al. 2013; Yao et al. 2008a).

Clinical manifestations are sometimes hormonal related, due to secretion by the tumour (i.e., gastrin, insulin, vasoactive intestinal peptide, etc). However, most of pNETs are discovered incidentally, or as a result of the mass effect of the primary tumour and distant metastases; these may be found at diagnosis in about half of the patients with nonfunctioning pNETs (Frilling et al. 2014; Halfdanarson et al. 2008; Ito et al. 2012a; Yao et al. 2008a).

pNETs generally show moderate biological aggressiveness and a slow rate of growth. Indeed, although 
surgical resection is the main therapeutic approach for functioning pNETs, some evidences support the safety of a conservative approach for asymptomatic sporadic nonfunctioning pNETs with a tumour size of $2 \mathrm{~cm}$ or less (Boninsegna et al. 2012; Guo and Wu 2013; Knigge and Hansen 2012).

Numerous factors for predicting survival have been identified, including age at diagnosis, functional status, Ki-67 index, and stage (Halfdanarson et al. 2008). Fiveyear survival rate is approximately $80-90 \%$ for localized pNETs, dropping to $\sim 40 \%$ for patients with metastatic disease (Cherenfant et al. 2013; Lawrence et al. 2011).

Treatment of patients with advanced/progressive pNETs is challenging and includes locoregional procedures to manage liver metastases and systemic therapies for diffuse metastatic disease with high and/or rapidly progressing tumour burden (Pavel et al. 2016). Systemic treatments encompass somatostatin analogs (SSAs), peptide receptor radionuclide therapy (PRRT), interferonalpha therapy, conventional chemotherapy, and targeted therapies.

In the last few years, the therapeutic approach for advanced pNETs has dramatically changed. Both sunitinib and everolimus, which target respectively multiple tyrosine kinase receptors and the mTOR signalling pathway, have been approved for the treatment of advanced pNETs (Raymond et al. 2011; Yao et al. 2011).

In the EU, everolimus is currently authorised for the "treatment of unresectable or metastatic, well- or moderately differentiated neuroendocrine tumours of pancreatic origin in adults with progressive disease" (http://www. ema.europa.eu/docs/en_GB/document_library/EPAR_-_ Product_Information/human/001038/WC500022814. pdf).

Promising efficacy has been shown for temozolomide, alone or combined with capecitabine (Ekeblad et al. 2007; Strosberg et al. 2011), and for radionuclide therapy with 177Lu-Dotatate (Strosberg et al. 2017). However, the optimal sequence of these different treatments has not been defined to date, because studies specifically designed for identifying which therapy is to be preferred for a specific moment of the disease course are lacking, and data from ongoing trials on this issue are still awaited.

With the aim to shed light on the treatment algorithm for advanced pNETs, we performed an up-to-date critical review taking into account the results of both retrospective and prospective published studies on everolimus as the first line therapy for pNETs, and the recommendations of widespread international guidelines on the clinical management of NETs. In addition, we performed an extensive search on the Clinical Trial Registries databases worldwide, to gather information on the ongoing clinical trials related to this specific topic.

\section{Molecular background}

\section{Activation of the mTOR pathway in pNETs}

The phosphatidylinositol 3-kinase(PI3K)-AKT pathway is a major mediator of the intracellular signalling network regulating essential cellular functions such as metabolism, proliferation, growth, and apoptosis (Altomare and Testa 2005). PI3K is recruited to the plasma membrane in response to extracellular signals, mainly growth factors (i.e., VEGF, PDGF, IGF-1, etc), which bind to specific cell membrane receptors thus activating the PI3K/AKT pathway cascade (Fig. 1).

The serine/threonine kinase mTOR (mammalian target of rapamycin) is the most important downstream component of the PI3K/AKT signalling pathway (Vignot et al. 2005). mTOR is constituted by two separate complexes: mTOR complex 1 (mTORC1) and complex 2 (mTORC2). Key functions of mTORC1 are largely identified, since it has been shown to essentially promote cell growth and proliferation and to be sensitive to rapamycin inhibitory action. mTORC2 role is less well defined: it is rapamycin insensitive (Zeng et al. 2007), and regulates actin cytoskeleton and cell migration (Jacinto et al. 2004).

The development of human cancers, including pNETs, may be a consequence of overexpressed extracellular signals or aberrant activity of cell membrane receptors and their related downstream signalling. For instance, a downregulation of PTEN and TSC2, both of which negatively regulate mTOR signalling, was reported in primary pNETs (Missiaglia et al. 2010). Somatic mutations of genes coding components of the PI3K/AKT/mTOR pathway have been reported in $15 \%$ of non-familial pNETs (Jiao et al. 2011). Activation of AKT was observed in 28 out of 46 NETs, thus suggesting a role of p-AKT in NET tumourigenesis (Ghayouri et al. 2010). The expression rates of both mTOR and activated mTOR (p-mTOR) ranged from 45.0 to $70.8 \%$ and from 44.4 to $61.8 \%$, respectively, in a retrospective series of NETs (Zhou et al. 2010). All these evidences document a relevant role of the mTOR signalling in the pathogenesis of pNETs.

\section{Everolimus and pNETs}

\section{Activity, efficacy, and safety}

Rapamycin has immunosuppressive functions, and the protein complex named RAFT (rapamycin and FKBP12 target), a mammalian homolog of the yeast TOR proteins (Heitman et al. 1991; Kunz et al. 1993), has been 


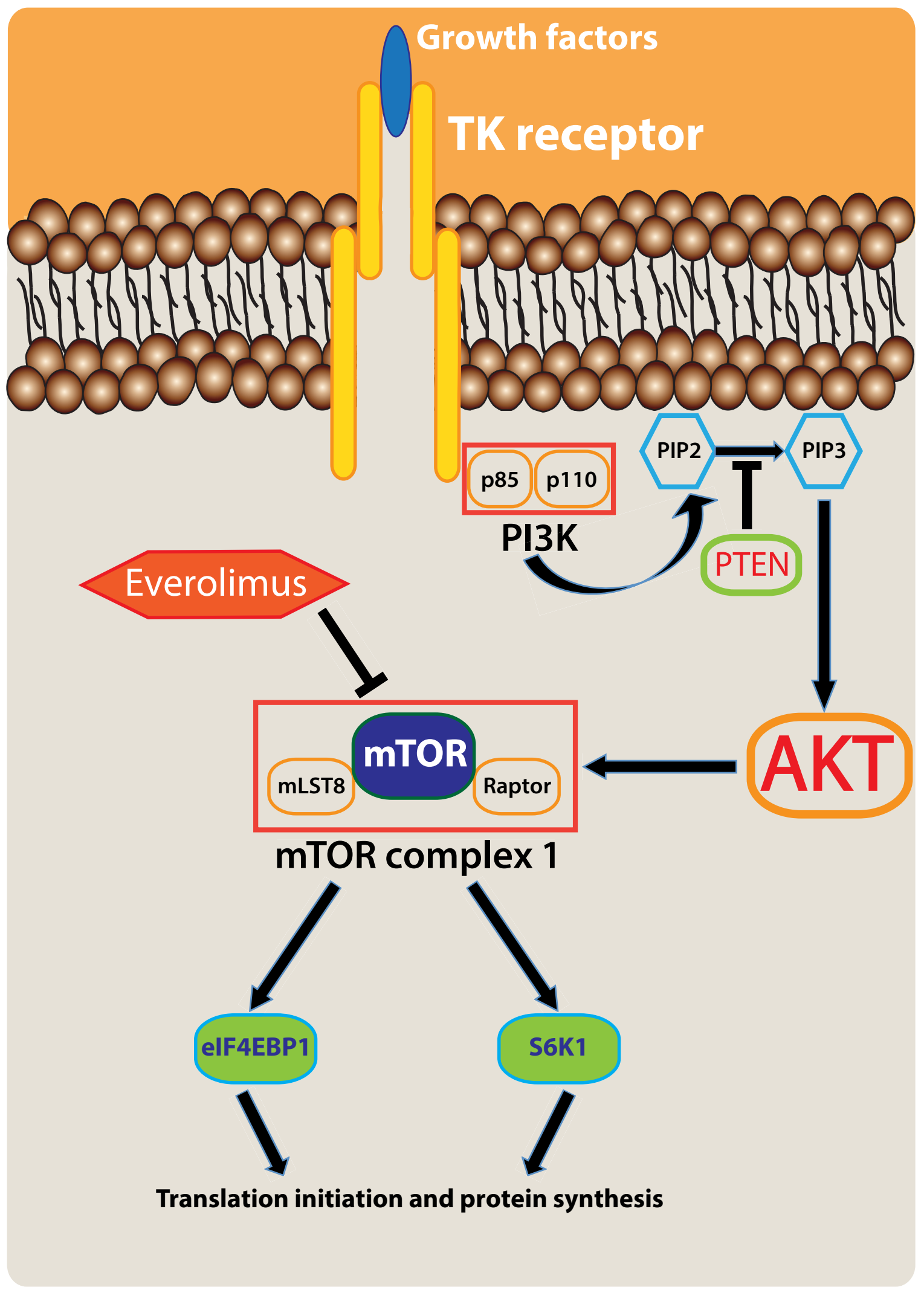

Fig. 1 Simplified representation of the PI3K-AKT-mTOR pathway and mechanism of action of everolimus 
suggested as its direct target in humans (Sabatini et al. 1994). Rapamycin and its analogs (i.e., everolimus) bind to an intracellular receptor, the FK506-binding protein (FKBP) (Harding et al. 1989; Siekierka et al. 1989), and this FKBP-rapamycin complex interacts with mTOR leading to inactivation of the mTOR signalling through dephosphorylation of both downstream effectors (4EBP1 and S6K1) of mTORC1 (Meric-Bernstam et al. 2012). mTOR signalling is implicated in cancer proliferation and progression (Osaki et al. 2004; Wullschleger et al. 2006), and several studies documented that inhibition of the PI3K/AKT/mTOR pathway can be helpful for the treatment of patients with cancer, including pNETs.

In vitro, the activity of everolimus on the modulation of cell proliferation was demonstrated in BON1 cells, a human pNET cell line characterized by constitutive activation of the PI3K/AKT/mTOR pathway. In this study Zitzmann and coworkers (Zitzmann et al. 2007) observed that treatment with everolimus led to inhibition of cell growth by G0/G1 cell cycle arrest and promotion of apoptosis. Similarly, this antiproliferative effect was observed in INS1 cells, a rat insulinoma cell line, where everolimus inhibited phosphorylation of both mTOR and its downstream target S6K1 (Grozinsky-Glasberg et al. 2008).

These evidences encouraged researchers to translate these findings into clinical settings. Yao and coworkers first demonstrated the efficacy and safety of everolimus in patients with advanced low- to intermediate-grade NETs (Yao et al. 2008b). They showed that everolimus in association with octreotide LAR was well tolerated and provided promising antitumour activity: among the 30 patients with islet cell tumours, partial response was obtained in $27 \%$ and median progression-free survival (PFS) was 50 weeks. Thereafter, a phase 2 study confirmed the effectiveness of treatment with everolimus in patients with metastatic pNETs who progressed after cytotoxic chemotherapy (Yao et al. 2010). On May 2011, the food and drug administration approved everolimus for the treatment of progressive pNETs, in case of unresectable locally advanced or metastatic disease. This approval was based on the findings obtained in a randomized controlled phase 3 trial that compared the treatment with everolimus $10 \mathrm{mg} / \mathrm{die}$ $(n=207)$ to placebo $(n=203)$ in patients with advanced pNETs (Yao et al. 2011). Investigators reported that treatment with everolimus was associated with a $65 \%$ reduction in the risk of progression or death. Ten (5\%) out of 207 patients receiving everolimus obtained an objective tumour response, the main antitumour activity of everolimus was related to a stabilization of disease ( $73 \%$ of cases), while some degree of tumour shrinkage was observed in $64 \%$.

Efficacy of everolimus has also been reported with extra-pancreatic NETs. A recent systematic review evaluated the efficacy of everolimus for extra-pNETs retrieving
22 studies, corresponding to 456 patients with NETs originating from several primary sites, including small bowel, lung, and colon/rectum (Faggiano et al. 2016). These findings were confirmed by a randomized, double-blind, placebo-controlled, phase 3 trial that investigated the efficacy and safety of everolimus in patients with non-functioning well-differentiated (G1 or G2) NETs of gastrointestinal $(n=175)$ or lung origin $(n=90)$. Treatment with everolimus reduced risk of progression by 44 and $50 \%$ in gastrointestinal and lung NETs, respectively (Yao et al. 2016).

All studies confirmed that everolimus was generally well tolerated, and grade 3-4 drug-related adverse events were not frequent. The most common grade 3-4 adverse events were stomatitis $(7 \%)$, anemia $(6 \%)$, and hyperglycaemia (5\%) (Yao et al. 2011). The drug-related toxicity profile is a main issue to consider when starting a new therapy line. For instance, compared to everolimus, octreotide and lanreotide are generally well tolerated. The most common adverse events in patients treated with SSAs are moderate and regard the gastrointestinal tract (diarrhoea, abdominal pain, and cholelithiasis). The frequency of grade 3-4 adverse events in patients treated with chemotherapy (i.e. streptozotocin, 5-FU, or doxorubicin), instead, is higher (about 20\%) than that observed for everolimus and usually includes hematologic, heart, and kidney toxicities (Valle et al. 2014).

Despite its well-established antitumour activity, treatment with everolimus is not effective in a subset of patients, possibly due either an innate or an acquired tumour resistance. Molecular events underlying resistance to everolimus are not completely known, but probably descend from the activation of compensatory feedback loops, and crosstalk between the PI3K/AKT/mTOR cascade and other pathways (Burris 2013; Markman et al. 2010). Given the presence of mechanisms of resistance, novel PI3K/AKT/mTOR inhibitors (Vandamme et al. 2016) and/or drugs with dual target inhibitory effects are currently under evaluation.

It should be emphasized that all the evidences about the efficacy of everolimus in pNETs come from patients with sporadic tumours, whereas the drug has not formally been evaluated in inherited disorders such as multiple endocrine neoplasia type 1 (MEN1) (Yates et al. 2015), and extrapolations from results obtained in patients with non-familial pNETs deserve caution.

Mutations in the mTOR pathway have been found in $\sim 15 \%$ of pNETs, making everolimus an attractive therapeutic option in this setting. Indeed, everolimus has been studied in the widest development program for a new drug in pNETs. A pathophysiological rationale for associating everolimus and SSAs has been hypothesized, since the upregulation of the IGF1 pathway has been proposed as a potential resistance mechanism for mTOR inhibitors. Furthermore, SSAs reduce serum IGF1 levels, which, in turn, 
seem to activate mTOR and to increase cell proliferation (O'Reilly et al. 2006; Pollak et al. 1989; von Wichert et al. 2000). From a clinical point of view, in the phase 2 openlabel, nonrandomized study RADIANT-1 (Yao et al. 2010), which was not designed to evaluate whether everolimus combined with SSAs was superior to everolimus monotherapy, the combined therapy resulted in a longer PFS (16.7 vs. 9.7 months in the everolimus monotherapy arm). This disease-stabilizing activity was confirmed in the randomized phase 3 clinical trial RADIANT-3 (Yao et al. 2011), which documented a significantly longer PFS in patients with pNETs randomized to the association of everolimus and octreotide (11 vs. 4.6 months in the octreotide alone arm). In addition, the combination of everolimus with lanreotide suggested efficacy in a retrospective cross-sectional analysis, without unexpected toxicities, apparently through a synergistic effect (Capdevila et al. 2015). The possibility of enhanced efficacy with SSAs combined with everolimus is actually being explored in randomized clinical trials, such as the phase 2 study COOPERATE- 2 and the LUNA clinical trial, whose definitive results are eagerly awaited, even if preliminary data are disappointing. However, in daily clinical practice, SSAs and everolimus are often given concurrently for patients with pNETs, especially functioning pNETS.

\section{Predictive factors of response to everolimus}

As cancer therapies are expensive and often associated with significant adverse events, identifying factors able to predict which patients will experience useful clinical responses is of high relevance to patients, clinicians, and health authorities.

Several studies investigated the role of various predictors of response to mTOR inhibitors, including clinical, biological, and histological factors (Zatelli et al. 2016).

Response to mTOR inhibitors has been associated with the expression levels of the mTOR pathway components, which have been evaluated by immunohistochemistry (IHC) and molecular studies on tissue specimens. Recently, effectiveness of everolimus was positively correlated to the IHC overexpression of phosphorylated p70S6K (Benslama et al. 2016). Response to mTOR inhibitors seems to correlate with the presence of mutations of genes involved in the PI3K/AKT/mTOR pathway (Meric-Bernstam et al. 2012). Sensitivity to rapamycin was related to genomic aberration of PIK3CA and/or PTEN (Meric-Bernstam et al. 2012), while resistance to everolimus has been observed in patients with oncogenic KRAS mutation (Di Nicolantonio et al. 2010). Recently, in patients with pNETs responsiveness to everolimus treatment has been correlated with a higher protein levels of the IGF1 downstream signalling involved in mTOR pathway (Falletta et al. 2016).
At present, however, IHC studies and assessment of mutational status of the mTOR signalling by DNA/protein evaluation are not routinely recommended to drive the selection of patients that may benefit from treatment with everolimus.

\section{Methodology for literature search strategy}

\section{Definition of first line}

Since available data come from studies on patients who frequently received SSAs prior, we considered "Everolimus as first line" in the following conditions: Everolimus given alone or in association with other therapies (including chemotherapy and SSAs), in patients who did not receive any previous therapies other than SSAs.

\section{Current knowledge}

Published retrospective and prospective studies Four investigators (M.G., P.M., G.F., and F.R.) independently searched the Medline database (via the PubMed interface) to identify potentially relevant articles on the therapeutic use of everolimus (alone or associated with other treatments) as a first line therapy for pNETs. The search was last updated February 15th, 2017. Only articles published in English language were considered. The search strategy included the following terms: "neuroendocrine tumour", "neuroendocrine carcinoma", "pancreatic neuroendocrine tumour", "pancreatic neuroendocrine carcinoma", "everolimus", and "RAD001".

Articles were considered without any restriction, and only Editorials and Letters were excluded. Single case reports were excluded and only studies describing two or more patients previously treated with everolimus for a pNET were considered. The selected abstracts were then further assessed for a full-text evaluation. Additional studies were identified by reviewing the references of all selected articles.

International guidelines Among the numerous available guidelines on the clinical management of NETs, issued by different scientific societies, four investigators (MG, P.M., G.F., and F.R) selected the 4 guidelines more frequently used at their centers for decision-making.

\section{Future perspectives}

Registered clinical trials (ReCTs) To detect all potentially relevant ReCTs on everolimus (alone or in association) as the first line therapy for pNETs, two investigators (G.F. and F.R.) independently searched the U.S. National Institutes of Health Registry (https://clinicaltrials.gov/), and all the "Primary Registries" defined according the WHO International 
Clinical Trial Registry Platform (ICTRP) (http://www. who.int/ictrp/network/en/) (see Table 1). The search was last updated February 15th, 2017. ReCTs published in all the Official Languages of the Primary Registries were considered. The terms included for the search strategy are the same used for the "Published Prospective Clinical Trials and Retrospective Studies". Only ReCTs for which the study protocol clearly states that enrolled patients are not receiving nor had previously received, at any time, any treatment for pNETs, including chemotherapy, radiation therapy, antibody based therapy, targeted therapy, biotherapy, etc., but excluding SSAs, were included. For the ReCTs in which the study protocol inclusion and exclusion criteria were not unambiguous, the Principal Investigator and/or the Sponsor were contacted for further clarifications.

\section{Results}

\section{Current knowledge}

\section{Published retrospective Studies}

Overall, 8 retrospective studies were identified (Bernard et al. 2013; Capdevila et al. 2015; Ferrer-Garcia et al. 2013; Fiebrich et al. 2011; Kulke et al. 2009; Liu et al. 2016; Panzuto et al. 2014; Tippeswamy et al. 2015), which included a total of 183 patients with pancreatic and 135 patients with extra-pancreatic NETs (see Table 2a).

Despite the methodological effort to specifically focus on studies on everolimus as the first line therapy, evaluation

Table 1 Primary registries of clinical trials defined according the WHO International Clinical Trial Registry Platform (ICTRP) (http:// www.who.int/ictrp/network/en/2

Australian New Zealand Clinical Trials Registry (ANZCTR)

Brazilian Clinical Trials Registry (ReBec)

Chinese Clinical Trial Registry (ChiCTR)

Clinical Research Information Service (CRiS) - Republic of Korea

Clinical Trials Registry-India (CTRI)

Cuban Public Registry of Clinical Trials (RPCEC)

EU Clinical Trials Register (EU-CTR)

German Clinical Trials Register (DRKS)

Iranian Registry of Clinical Trials (IRCT)

Japan Primary Registries Network (JPRN)

ISRCTN Registry ${ }^{\#}$

Thai Clinical Trials Registry (TCTR)—Thailand

The Netherlands National Trial Register (NTR)

Pan African Clinical Trial Registry (PACTR)

Sri Lanka Clinical Trials Registry (SLCTR)

\# Primary Clinical Trial Registry recognized by WHO and International Committee of Medical Journal Editors of efficacy of everolimus as the first line approach was possible in only $56(17.6 \%)$ patients with pNETs of the studies identified. Most patients were treated with everolimus as a second line drug, after failure of the previous medical treatments. Taking into account this limitation, treatment with everolimus led to disease stabilization in more than half of patients (Liu et al. 2016; Tippeswamy et al. 2015), and disappearance of hypoglycaemic symptoms in malignant insulinomas (Fiebrich et al. 2011).

\section{Published prospective studies}

Only two prospective studies aimed to assess the efficacy of everolimus as the first line for patients with pNETs, namely the RADIANT- 3 and the ITMO group study (see Table $2 \mathrm{~b}$ ).

Among the patients included in the RADIANT-3, 204 patients (103 in the everolimus arm and 101 in the placebo arm) were chemotherapy-naïve, and 206 patients (104 in the everolimus arm and 102 in the placebo arm) had been previously treated with chemotherapy (Yao et al. 2011).

A subgroup analysis of RADIANT-3 was performed aiming to assess the role of chemotherapy on the efficacy of everolimus (Lombard-Bohas et al. 2015). This subanalysis demonstrated no relevant difference of the efficacy of everolimus between patients previously treated with chemotherapy and those which were chemotherapy-naïve, according to the evaluation of local investigators as well as central reviewers. In particular, the median PFS in chemonaïve patients who received everolimus was 11.4 months for local investigators and 14 months for central reviewers, whereas in non chemotherapy-naïve patients who received everolimus was 11 months for local investigators and 11.4 months for central reviewers. The objective response rate (ORR) and disease control rate (DCR) showed no significant differences between the two groups. Therefore, data obtained from the RADIANT-3 suggest that everolimus is effective in patients with advanced, well, and moderately differentiated pNETs, both before and after chemotherapy.

The ITMO group study was focused on the use of everolimus as the first line in a patient population with NETs (Bajetta et al. 2014). Fifty patients with lung and gastroenteropancreatic (GEP) NETs were studied. Thirteen of them had carcinoid syndrome, while 14 patients had a pNET. None of the patients previously received any treatment. During the study, patients were treated with everolimus $10 \mathrm{mg} /$ day and octreotide $30 \mathrm{mg} / 28$ days. The ORR, which was the primary endpoint of the study, was $18 \%$ in the intent-to-treat population (ITT), formed by patients who received at least one dose of everolimus and $19.6 \%$ in the per-protocol population (PP). Four patients were excluded from PP analysis, one for major breach of the protocol and three because they had not reached the minimum 


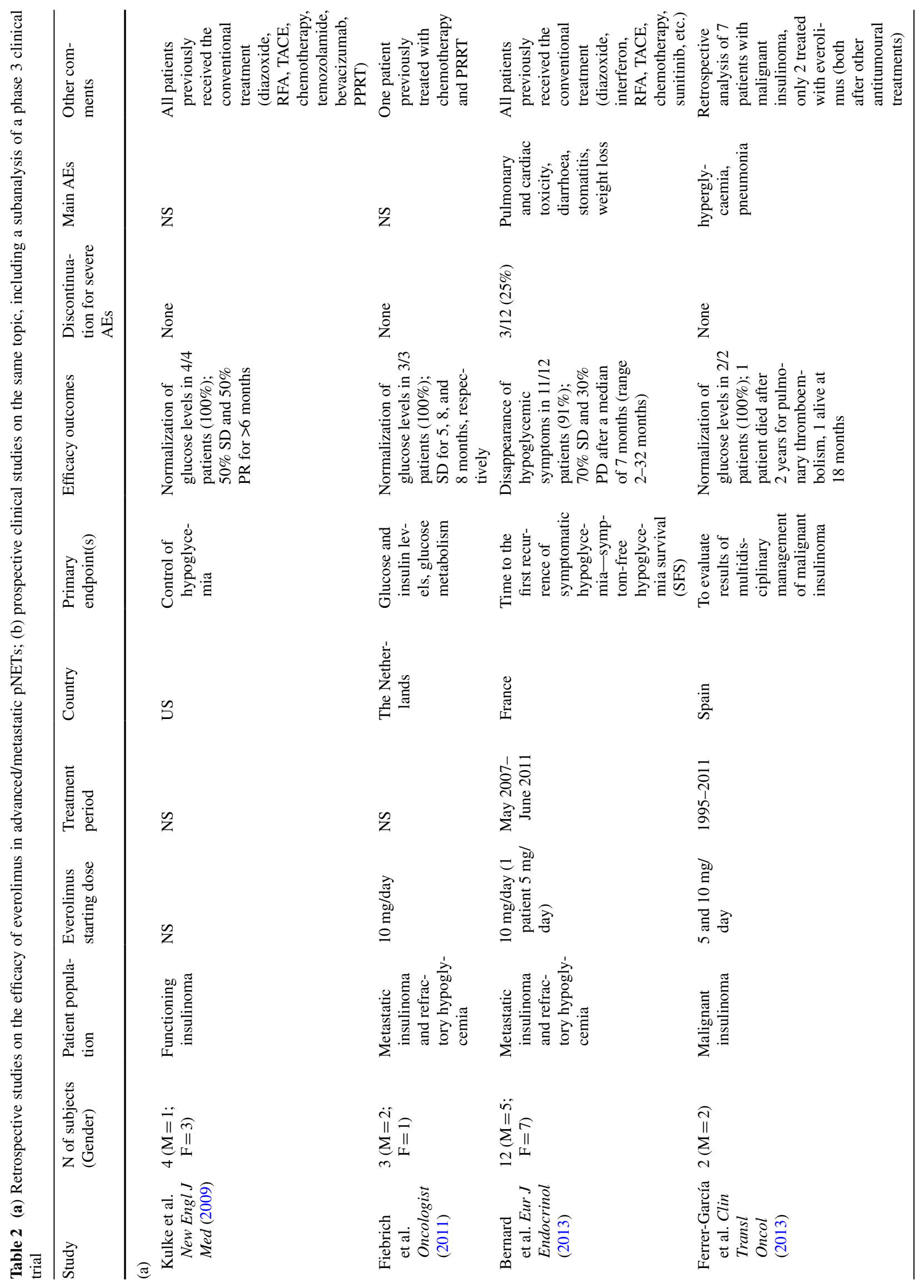




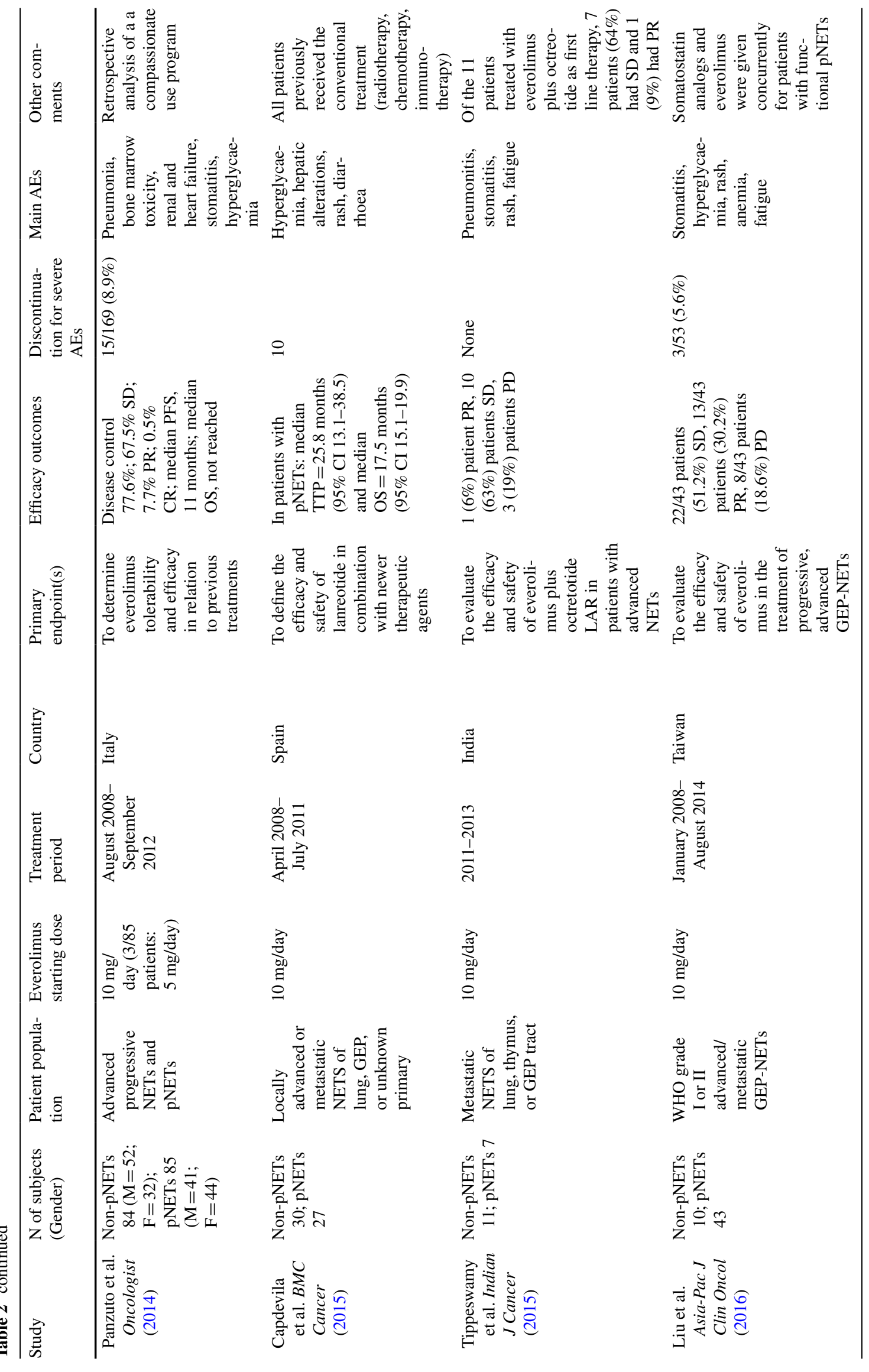




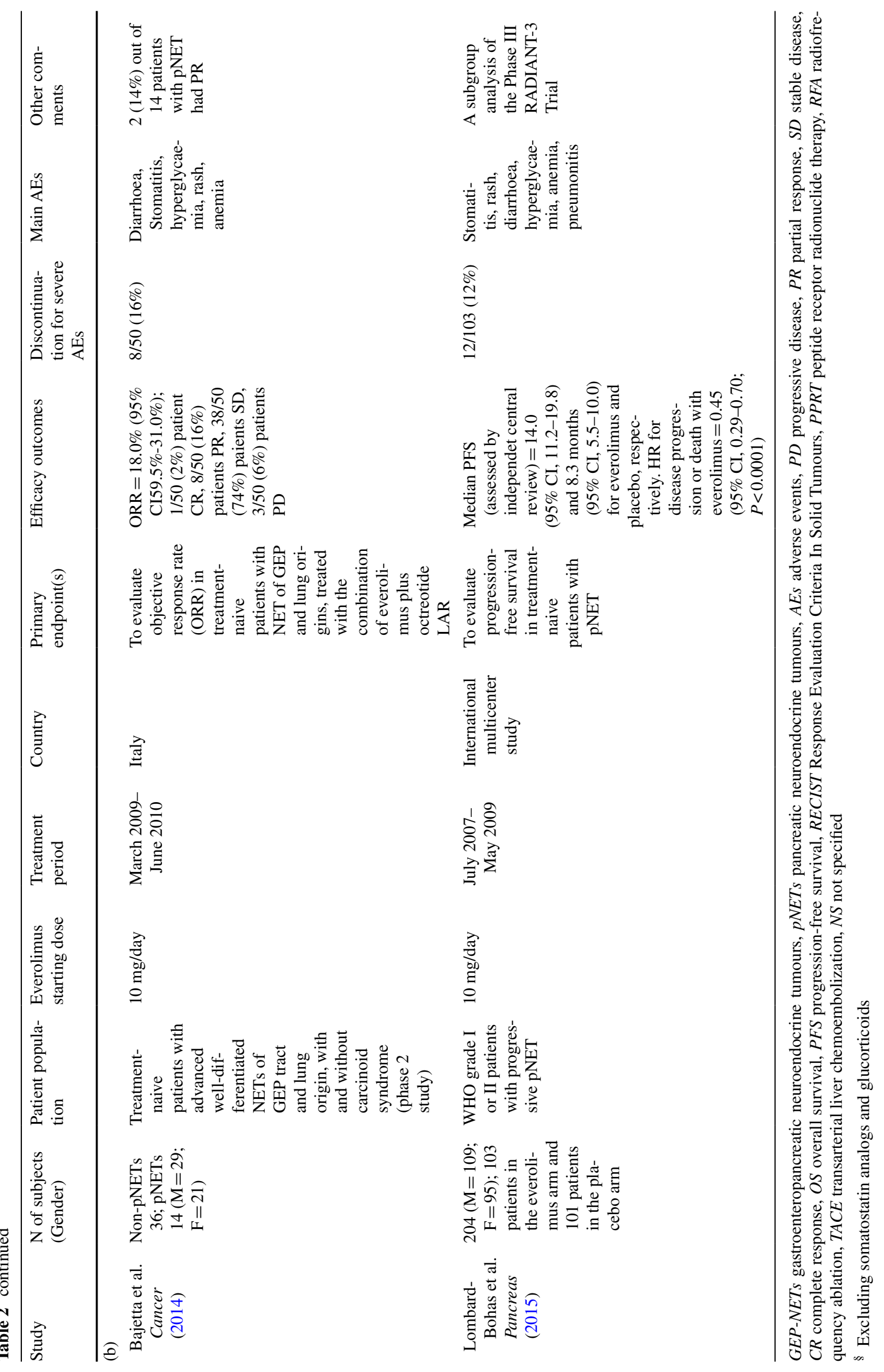


established dose of everolimus. The analysis was not specified for patient subgroups according to the site of origin of the NET, but the authors stated that no statistically significant differences in ORR were found for different locations of NETs. They also specified that, among patients with pNETs, 2 partial responses (PR) were observed, while none showed a complete response (CR). As for the secondary endpoints [Time To Progression (TTP), and Overall Survival (OS)], no significant differences were observed after a median of 227 days (Bajetta et al. 2014). Even with the limitations of the study, such as the low number of subjects and the lack of a control group, the ITMO study Group suggests that combined therapy with everolimus/octreotide LAR can be effective as first line for patients with pNETs as well as with NETs of other origin.

\section{International guidelines}

To the purpose of this review, guidelines from the following scientific societies were considered: ESMO (European Society for Medical Oncology), ENETS (European Neuroendocrine Tumour Society), NANETS (North American Neuroendocrine Tumour Society), and NCCN (National Comprehensive Cancer Network).

In 2012, immediately after the publication of the RADIANT-3 trial, the ESMO updated its guidelines for GEPNETs (Oberg et al. 2012). Everolimus was mentioned as "another specific therapy" in the paragraph dedicated to the medical therapy for the management of advanced/metastatic disease, currently registered for treatment of pancreatic NETs worldwide, to be used alone or in combination with a SSA. Indeed, SSAs were designed as "the recommended the first line therapy in non-functioning as well as functioning progressive G1/G2 NETs". Browsing through the ESMO treatment algorithm, however, everolimus appears as the first line therapy for G1/G2 (Ki-67: 2-20\%) non-functioning pNETs (just like sunitinib), whereasamong functioning pNETs-its first line use is hypothesized for symptomatic treatment of insulinomas.

The 2010 edition of the NANETS consensus guidelines for the management of NETs simply mentioned everolimus as a drug that may control hypoglycaemia in patients with metastatic insulinomas (Kulke et al. 2010). Three years later, the updated version of NANETS guidelines had to take into account the publication of "a number of practicechanging studies", namely the completion of several phase 3 trials evaluating octreotide, sunitinib, and everolimus. In the new document, indication for initiating targeted therapies (or cytotoxic chemotherapy) in patients with advanced pNETs was defined "a controversial topic in the management of NETs". However, dealing with the management of advanced pNETs, everolimus (like sunitinib, hepatic artery embolization, or simple observation for a brief 3-month period) was defined as a treatment to be considered in newly diagnosed patients with high-volume disease. Furthermore, therapy with everolimus was defined to be "recommended" in the event of progressive disease, and for hormonal syndrome control of insulinomas. According to the NANETS guidelines, however, lines of therapy for pNETs have not been definitely established, and "the proposed order of listing does not imply order of therapy" (Kunz et al. 2013).

Currently available NCCN guidelines for the management of well-differentiated (Grade 1-2) pNETs were issued in 2016. According to these guidelines, everolimus (with or without SSAs) should be considered as first line therapy only in the management of locoregional unresectable disease and/or distant metastases in patients with: (1) symptomatic disease; or (2) clinically significant tumour burden; or (3) clinically significant progressive disease (NCCN 2016). However, in the absence of prospective randomized trials, there is no clear recommendation for preferring everolimus as the first line choice over other choices such as sunitinib or cytotoxic chemotherapy or hepatic regional therapy or cytoreductive surgery/ablative therapy.

In the 2012 consensus guidelines from the ENETS, everolimus was mentioned as an option after failure of chemotherapy in pNETs, being considered as the first line therapy in exceptional cases as an alternative to locoregional therapies or chemotherapy (e.g., symptomatic, bulky disease or intolerance of ongoing therapy) (Falconi et al. 2012). The panel did not recommend an early unselected use of the drug due to the lack of long-term toxicity data. Furthermore, everolimus was also suggested for the treatment approach to liver metastases from pNETs, as an alternative to SSAs, chemotherapy, sunitinib, or PRRT (Pavel et al. 2012). Conversely, according to the last version of the ENETS guidelines for the management of distant metastatic disease of NETs, everolimus (and sunitinib) can be considered a first line systemic, antiproliferative therapy for advanced and/or progressive non-functional G1/G2 pNETs, representing one of the different treatment alternatives, especially if SSAs are not an option, and if systemic chemotherapy is not feasible, not clinically required, or not tolerated (Pavel et al. 2016).

\section{Future perspectives}

Overall, $128 \mathrm{ReCTs}$ were identified. Of the $128 \mathrm{ReCTs}$ analyzed, only 4 matched the initial requirements, therefore, specifically dealing with the topic "Everolimus as first line therapy on pNETs".

In brief, we detected 4 Phase 2 studies, and everolimus is being employed: (1) alone; (2) in combination with octreotide and metformin; (3) in combination with temozolomide; and (4) in combination with cisplatinum. Of the 
4 ReCTs, 3 are still recruiting participants at the time of manuscript writing. Of the 5 ReCTs, 1 is not yet open for participant recruitment at the time of manuscript writing, and 3 are still recruiting participants. Details of the ReCTs identified are summarized in Table 3.

\section{Discussion}

Systemic therapeutic options for pNETs have dramatically increased during the past decade, now including SSAs and PRRT, targeted therapies such as everolimus and sunitinib, and the newly tested cytotoxic agents (i.e., capecitabine alone or in combination with temozolamide). These options can be variously employed together with surgery, locoregional treatments (e.g., radiofrequency ablation, cryoablation, chemoembolization, and radioembolization), and/ or other drugs (e.g., diazoxide for insulinomas and proton pump inhibitors for gastrinomas), in a multimodal setting.

Everolimus, by targeting the mTOR pathway, provides a valid rationale for treating unresectable malignant NETs. In particular, both data derived from phase 3 trials and from the real-world setting witness that everolimus is effective and safe enough for the treatment of advanced, progressive G1 and G2 pNETs (Panzuto et al. 2014; Yao et al. 2010, 2011).

However, data on pNETs therapeutic sequence are limited and clinicians have to take management decisions based on their own experience and/or on expert recommendations. Consequently, the best place of everolimus in the therapeutic algorithm for advanced pNETs is still unknown.

Collectively, existing guidelines on the clinical management of pNETs provide no definitive recommendations on the most suited position of everolimus in the treatment algorithm for advanced pNETs, apparently placing the drug randomly as an alternative to other choices. Similarly, according to a recently published consensus article on the appropriateness of a variety of systemic treatments in patients with pNETs, everolimus, sunitinib, and cytotoxic chemotherapy were all defined as appropriated as the first line therapy in patients with hormonally functioning or progressive tumours, without significant differences in ratings (Strosberg et al. 2015).

As far as efficacy of everolimus in patients naïve to cytotoxic chemotherapy is concerned, data arising from prospective studies on the use of everolimus as first line are few. The subanalysis of the patients enrolled in the RADIANT-3 trial (Lombard-Bohas et al. 2015) showed similar efficacy in patients not chemonaïve and in patients chemonaïve, with a significant prolonged median PFS in both subgroups, and without any differences both in objective response and in disease control rate. However, the proportion of patients who developed grade 3-4 thrombocytopenia was higher in the prior chemotherapy group. Indeed, treatment duration with everolimus was longer for patients who were naïve to chemotherapy, thus suggesting that it was better tolerated in the chemonaïve group. The ITMO study group (Bajetta et al. 2014) suggests that combined therapy with everolimus/octreotide LAR can be effective as the first line for patients with NETs. The two studies are obviously not comparable, both for the different sample size and for the design of the study. The population of RADIANT 3 was large, with a long duration of disease: about $60 \%$ of the patients of the two arms had a duration of disease $>2$ years. In addition, the population of patients was heterogeneous in respect to the previous treatments. In the everolimus group, 23\% of the patients had received radiotherapy, 49\% SSAs, and 50\% chemotherapy. During the study, best supportive care was also permitted, which also included SSAs in $40 \%$ of patients. Patients with pNETs in the ITMO study group were only 14 , recently diagnosed. None of them had received other medical therapies prior to the study, and in all cases, everolimus was associated with octreotide LAR.

Panzuto et al. (2014) performed a retrospective study on daily clinical practice (including 85 pNETs, 66 of whom with G2 pNETs) to determine everolimus tolerability and efficacy in relation to the previous treatments. All patients had previously been treated with SSAs, PRRT, interferon, and/or systemic chemotherapy. Everolimus was associated with SSAs in $87 \%$ of patients. Higher severe toxicity occurred in patients previously treated with systemic chemotherapy and/or PRRT, with a 12-fold increased risk for grade 3-4 adverse events in patients pre-treated with both chemotherapy and PRRT. The most frequent severe adverse events in this setting were haematological toxicity, renal failure, and peripheral oedema. According to authors, their findings should raise the issue of planning treatment with everolimus before other options, prompting the use of particular caution in the use of everolimus in heavily pretreated subjects. Conversely, a retrospective analysis by Kamp et al. (2013) on 24 patients treated with everolimus showed that the safety profile of the drug was not influenced by the previous PRRT with 177Lu-octreotate (Kamp et al. 2013).

Even if everolimus in association with octreotide has been shown to improve PFS regardless of previous SSA exposure, patients who were naïve to SSAs experienced greater benefit from this association, according to a retrospective subset analysis of patients with advanced NETs in the RADIANT-2 study (Anthony et al. 2015; Shah et al. 2011; Yao et al. 2011). These findings suggest that the effectiveness of everolimus could be maximized in previously untreated patients, perhaps, because they have not developed partial or complete resistance to SSAs, yet. 


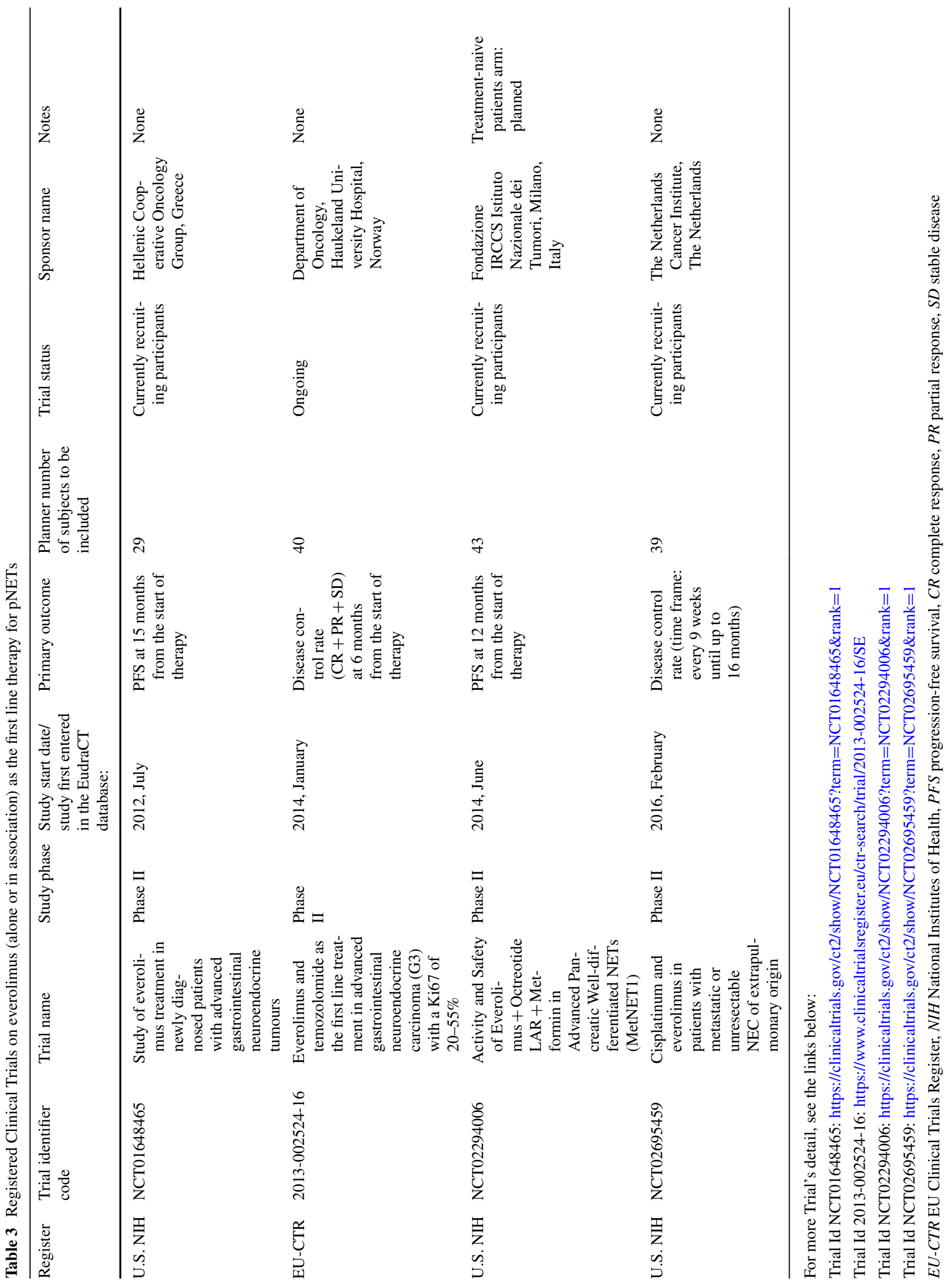


However, prospective data on everolimus as the first line therapy for pNETs, before and instead of SSAs, are too scarce to recommend this option.

The possibility of enhanced efficacy with SSAs combined with everolimus has recently been explored in two randomized clinical trials, the phase 2 study COOPERATE-2 (available at http://www.karger.com/Article/ Pdf/431385) and the LUNA clinical trial (available at http://annonc.oxfordjournals.org/content/27/suppl_6.toc). Preliminary results from these studies documented that combining everolimus and SSAs is not superior to everolimus alone in progressive pNETs and progressive carcinoids of lung/thymus, with respect to prolongation of PFS.

Collectively, these findings seem to suggest a potential for the first line therapy with everolimus in patients with unresectable, well-differentiated, advanced pNETs.

However, these findings derive from a few studies on patients treated and followed-up with different protocols. Moreover, since these studies were not designed to evaluate the efficacy of everolimus first line, extrapolating any data on chemotherapy-naïve patients with pNETs may lead to biased conclusions.

Our systematic analysis of ReCTs on "Everolimus as first line" offers, for the first time in literature, an updated summary about the upcoming clinical trials.

This overview (hopefully) offers the Clinician a "glance into the future" about the expected effects of everolimus (and of the drugs used in combination with everolimus) in the context of the medical conditions presently under investigation (advanced/metastatic/unresectable pNETs). Moreover, the awareness of the extremely limited number of ongoing studies in this field (that are, additionally, almost all in phase 2), may encourage researchers to address new studies in this uncharted area.

Everolimus, beyond exerting direct antiproliferative effects and stimulating NETs regression, may decrease insulin production and release, while inducing peripheral insulin resistance, with hyperglycaemia as a frequently observed side effect of this therapy. Conversely, patients with malignant unresectable insulinomas may take great advantage of this effect, as shown by some retrospective studies in this setting (Bernard et al. 2013; Ferrer-Garcia et al. 2013). Therefore, everolimus could be employed as first line treatment for progressive malignant insulinomas with refractory hypoglycaemia, as suggested by recent guidelines.

Furthermore, recently published observational data showed a better survival outcome in an Asian cohort of patients with progressive advanced GEP-NETs, most of pancreatic origin, if compared to the results of RADIANT-3 trial or other international experiences, but quite similar to a subgroup analysis of Japanese patients of the RADIANT-3 trial itself (Ito et al. 2012b; Jiao et al. 2011; Liu et al. 2016). Indeed, also in the RADIANT-4 study,
Asian patients showed a better PFS than Caucasian population (Yao et al. 2016). It has been speculated that this ethnic disparity in tumour response rate can, at least in part, be explained by a higher frequency of activating mutations in the mTOR pathway in pNETs from Asian patients (Jiao et al. 2011; Yuan et al. 2014). In the same study, a trend toward a longer overall survival was observed in patients with liver metastases burden $<10 \%$ receiving everolimus (Liu et al. 2016). Therefore, Asian patients with non-functioning pNETs or with malignant insulinomas, but with a limited metastatic burden to the liver, could represent the ideal setting for performing prospective trials comparing the efficacy of everolimus as the first line in this setting, with respect to other drugs.

Finally, treatment strategies for advanced/metastatic pNETs clearly depend not only on the stage, grading, functional status, the variable clinical course, and the local availability of different alternatives (e.g., locoregional treatment skills or the opportunity to use PRRT), but also-and ultimately—on costs.

\section{Conclusions}

Our critical review confirms the lack of high-quality data to recommend everolimus as the first line therapy for pNETs. The ongoing clinical trials reported in this review will hopefully help clinicians, in the near future, to better evaluate the role of everolimus as the first line therapy for pNETs. Besides, further randomized clinical trials will be required to confirm the promising results recently described by pilot studies, or derived by retrospective studies and subgroup analyses. However, there is already enough evidence to recommend everolimus as the first line therapy for patients with symptomatic malignant unresectable insulin-secreting pNETs, to control the endocrine syndrome regardless of tumour growth.

Acknowledgements This review is part of the 'NIKE' project (Neuroendocrine tumours Innovation Knowledge and Education) led by Prof Annamaria Colao, which aims at increasing the knowledge on NETs. We would like to acknowledge all the Collaborators of this project: Manuela Albertelli, Vito Amoroso, Emanuela Arvat, Giuseppe Badalamenti, Emilio Bajetta, Roberto Baldelli, Antonio Bianchi, Gerardo Botti, Roberto Buzzoni, Davide Campana, Carlo Carnaghi, Giacomo Cartenì, Sara Cingarlini, Luisa Circelli, Vittorio Colantuoni, Jorgelina Coppa, Francesco Corcione, Maria Vittoria Dav1', Gaetano De Rosa, Ettore Degli Uberti, Marialaura Del Basso De Caro, Antonella Di Sarno, Massimo Falconi, Piero Ferolla, Diego Ferone, Francesco Fiore, Germano Gaudenzi, Carla Giordano, Dario Giuffrida, Erika Maria Grossrubatscher, Elia Guadagno, Valentina Guarnotta, Francesco Izzo, Andrea Lania, Secondo Lastoria, Silvana Leo, Fabio Lo Calzo, Francesco Logoluso, Marco Manzoni, Massimo Marchetti, Chiara Martini, Erika Messina, Massimo Milione, Roberta Modica, Vincenzo Montesarchio, Pietro Muto, Oscar Nappi, Elena Nazzari, Francesco Panzuto, Anna Pia, Alessandro Piovesan, 
Genoveffa Pizza, Alfredo Pontecorvi, Sara Pusceddu, Paola Razzore, Ferdinando Riccardi, Guido Rindi, Concetta Sciammarella, Francesca Spada, Salvatore Tafuto, Annibale Versari, Giovanni Vitale, and Maria Chiara Zatelli.

\section{Compliance with ethical standards}

Conflict of interest The authors declare that there is no conflict of interest that could be perceived as prejudicing the impartiality of the research reported.

Ethical approval This article does not contain any studies with human participants or animals performed by any of the authors.

\section{References}

Altomare DA, Testa JR (2005) Perturbations of the AKT signaling pathway in human cancer. Oncogene 24:7455-7464. doi:10.1038/sj.onc. 1209085

Anthony LB et al (2015) Impact of previous somatostatin analogue use on the activity of everolimus in patients with advanced neuroendocrine tumors: analysis from the phase III RADIANT-2 Trial. Neuroendocrinology 102:18-25. doi:10.1159/000381715

Bajetta E et al (2014) Everolimus in combination with octreotide long-acting repeatable in a first-line setting for patients with neuroendocrine tumors: an ITMO group study. Cancer 120:24572463. doi: $10.1002 / \mathrm{cncr} .28726$

Benslama $\mathrm{N}$ et al (2016) Prediction of response to everolimus in neuroendocrine tumors: evaluation of clinical, biological and histological factors. Invest New Drugs doi:10.1007/s10637-016-0363-6

Bernard V et al (2013) Efficacy of everolimus in patients with metastatic insulinoma and refractory hypoglycemia. Eur J Endocrinol 168:665-674 doi:10.1530/EJE-12-1101

Boninsegna L et al (2012) Malignant pancreatic neuroendocrine tumour: lymph node ratio and Ki67 are predictors of recurrence after curative resections. Eur J Cancer 48:1608-1615 doi:10.1016/j.ejca.2011.10.030

Burris HA, 3rd (2013) Overcoming acquired resistance to anticancer therapy: focus on the PI3K/AKT/mTOR pathway. Cancer Chemother Pharmacol 71:829-842 doi:10.1007/s00280-012-2043-3

Capdevila J et al (2015) Evaluation of the efficacy and safety of lanreotide in combination with targeted therapies in patients with neuroendocrine tumours in clinical practice: a retrospective cross-sectional analysis. BMC Cancer 15:495. doi:10.1186/ s12885-015-1512-6

Capelli P, Fassan M, Scarpa A (2012) Pathology—grading and staging of GEP-NETs. Best Pract Res Clin Gastroenterol 26:705717 doi:10.1016/j.bpg.2013.01.003

Cherenfant J et al. (2013) Predicting aggressive behavior in nonfunctioning pancreatic neuroendocrine tumors Surgery 154:785-791. doi:10.1016/j.surg.2013.07.004 (discussion 791-783)

Di Nicolantonio F et al (2010) Deregulation of the PI3K and KRAS signaling pathways in human cancer cells determines their response to everolimus. J Clin Invest 120:2858-2866 doi:10.1172/JCI37539

Ekeblad S et al (2007) Temozolomide as monotherapy is effective in treatment of advanced malignant neuroendocrine tumors. Clin Cancer Res 13:2986-2991. doi:10.1158/1078-0432.CCR-06-2053

Faggiano A et al (2016) Efficacy and safety of everolimus in extrapancreatic neuroendocrine tumor: a comprehensive review of literature. Oncologist 21:875-886 doi:10.1634/ theoncologist.2015-0420
Falconi M et al (2012) ENETS Consensus Guidelines for the management of patients with digestive neuroendocrine neoplasms of the digestive system: well-differentiated pancreatic non-functioning tumors. Neuroendocrinology 95:120-134. doi: $10.1159 / 000335587$

Falletta S et al (2016) mTOR inhibitors response and mTOR pathway in pancreatic neuroendocrine tumors. Endocr Relat Cancer 23:883-891 doi:10.1530/ERC-16-0329

Ferrer-Garcia JC et al (2013) Management of malignant insulinoma. Clin Transl Oncol 15:725-731 doi:10.1007/ s12094-012-0996-7

Fiebrich HB, Siemerink EJ, Brouwers AH, Links TP, Remkes WS, Hospers GA, de Vries EG (2011) Everolimus induces rapid plasma glucose normalization in insulinoma patients by effects on tumor as well as normal tissues. Oncologist 16:783-787 doi:10.1634/theoncologist.2010-0222

Frilling A et al (2014) Recommendations for management of patients with neuroendocrine liver metastases. Lancet Oncol 15:e8-e21. doi:10.1016/S1470-2045(13)70362-0

Ghayouri M, Boulware D, Nasir A, Strosberg J, Kvols L, Coppola D (2010) Activation of the serine/theronine protein kinase Akt in enteropancreatic neuroendocrine tumors. Anticancer Res 30:5063-5067

Grozinsky-Glasberg S et al (2008) Octreotide and the mTOR inhibitor RAD001 (everolimus) block proliferation and interact with the Akt-mTOR-p70S6K pathway in a neuro-endocrine tumour cell Line. Neuroendocrinology 87:168-181. doi:10.1159/000111501

Guo Q, Wu Y (2013) Surgical treatment of pancreatic islet cell tumor: report of 44 cases. Hepatogastroenterology 60:2099-2102

Halfdanarson TR, Rabe KG, Rubin J, Petersen GM (2008) Pancreatic neuroendocrine tumors (PNETs): incidence, prognosis recent trend toward improved survival. Ann Oncol 19:1727-1733. doi:10.1093/annonc/mdn351

Harding MW, Galat A, Uehling DE, Schreiber SL (1989) A receptor for the immunosuppressant FK506 is a cis-trans peptidyl-prolyl isomerase. Nature 341:758-760. doi:10.1038/341758a0

Heitman J, Movva NR, Hall MN (1991) Targets for cell cycle arrest by the immunosuppressant rapamycin in yeast. Science 253:905-909

Ito T, Igarashi H, Jensen RT (2012a) Pancreatic neuroendocrine tumors: clinical features, diagnosis and medical treatment: advances. Best Pract Res Clin Gastroenterol 26:737-753 doi:10.1016/j.bpg.2012.12.003

Ito $\mathrm{T}$ et al (2012b) Everolimus for advanced pancreatic neuroendocrine tumours: a subgroup analysis evaluating Japanese patients in the RADIANT-3 trial. Jpn J Clin Oncol 42:903-911 doi:10.1093/jjco/hys123

Jacinto E, Loewith R, Schmidt A, Lin S, Ruegg MA, Hall A, Hall MN (2004) Mammalian TOR complex 2 controls the actin cytoskeleton and is rapamycin insensitive. Nat Cell Biol 6:1122-1128 doi:10.1038/ncb1183

Jiao Y et al (2011) DAXX/ATRX, MEN1, and mTOR pathway genes are frequently altered in pancreatic neuroendocrine tumors. Science 331:1199-1203. doi:10.1126/science.1200609

Kamp K, Gumz B, Feelders RA, Kwekkeboom DJ, Kaltsas G, Costa FP, de Herder WW (2013) Safety and efficacy of everolimus in gastrointestinal and pancreatic neuroendocrine tumors after (177)Lu-octreotate. Endocr Relat Cancer 20:825-831 doi:10.1530/ERC-13-0254

Knigge U, Hansen CP (2012) Surgery for GEP-NETs. Best Pract Res Clin Gastroenterol 26:819-831 doi:10.1016/j.bpg.2012.12.005

Kulke MH, Bergsland EK, Yao JC (2009) Glycemic control in patients with insulinoma treated with everolimus. N Engl J Med 360:195-197. doi:10.1056/NEJMc0806740 
Kulke MH et al (2010) NANETS treatment guidelines: well-differentiated neuroendocrine tumors of the stomach and pancreas. Pancreas 39:735-752. doi:10.1097/MPA.0b013e3181ebb168

Kunz J, Henriquez R, Schneider U, Deuter-Reinhard M, Movva NR, Hall MN (1993) Target of rapamycin in yeast, TOR2, is an essential phosphatidylinositol kinase homolog required for G1 progression. Cell 73:585-596

Kunz PL et al (2013) Consensus guidelines for the management and treatment of neuroendocrine tumors. Pancreas 42:557-577. doi:10.1097/MPA.0b013e31828e34a4

Lawrence B, Gustafsson BI, Chan A, Svejda B, Kidd M, Modlin IM (2011) The epidemiology of gastroenteropancreatic neuroendocrine tumors. Endocrinol Metab Clin North Am 40:1-18. doi:10.1016/j.ecl.2010.12.005 (vii)

Liu CT et al (2016) The efficacy and safety of everolimus for the treatment of progressive gastroenteropancreatic neuroendocrine tumors: A multi-institution observational study in Taiwan. Asia Pac J Clin Oncol doi:10.1111/ajco.12571

Lombard-Bohas C et al (2015) Impact of prior chemotherapy use on the efficacy of everolimus in patients with advanced pancreatic neuroendocrine tumors: a subgroup analysis of the phase III RADIANT-3 trial. Pancreas 44:181-189. doi:10.1097/ MPA.0000000000000262

Markman B, Dienstmann R, Tabernero J (2010) Targeting the PI3K/ Akt/mTOR pathway-beyond rapalogs. Oncotarget 1:530-543. doi:10.18632/oncotarget.101012

Meric-Bernstam F et al (2012) PIK3CA/PTEN mutations and Akt activation as markers of sensitivity to allosteric mTOR inhibitors. Clin Cancer Res 18:1777-1789. doi:10.1158/1078-0432.CCR-11-2123

Missiaglia E et al (2010) Pancreatic endocrine tumors: expression profiling evidences a role for AKT-mTOR pathway. J Clin Oncol 28:245-255. doi:10.1200/JCO.2008.21.5988

O'Reilly KE et al (2006) mTOR inhibition induces upstream receptor tyrosine kinase signaling and activates Akt. Cancer Res 66:1500-1508. doi:10.1158/0008-5472.CAN-05-2925

Oberg K, Knigge U, Kwekkeboom D, Perren A, Group EGW (2012) Neuroendocrine gastro-entero-pancreatic tumors: ESMO Clinical Practice Guidelines for diagnosis, treatment and follow-up. Ann Oncol 23(Suppl 7):vii124-130. doi:10.1093/annonc/mds295

Osaki M, Oshimura M, Ito H (2004) PI3K-Akt pathway: its functions and alterations in human cancer. Apoptosis 9:667-676. doi:10.1023/B:APPT.0000045801.15585.dd

Panzuto F et al (2014) Real-world study of everolimus in advanced progressive neuroendocrine tumors. Oncologist 19:966-974. doi:10.1634/theoncologist.2014-0037

Pavel M et al (2012) ENETS Consensus Guidelines for the management of patients with liver and other distant metastases from neuroendocrine neoplasms of foregut, midgut, hindgut, and unknown primary. Neuroendocrinology 95:157-176. doi:10.1159/000335597

Pavel M et al (2016) ENETS consensus guidelines update for the management of distant metastatic disease of intestinal, pancreatic, bronchial neuroendocrine neoplasms (NEN) and NEN of unknown primary site. Neuroendocrinology 103:172-185. doi:10.1159/000443167

Pollak MN, Polychronakos C, Guyda H (1989) Somatostatin analogue SMS 201-995 reduces serum IGF-I levels in patients with neoplasms potentially dependent on IGF-I. Anticancer Res 9:889-891

Raymond E et al (2011) Sunitinib malate for the treatment of pancreatic neuroendocrine tumors. New Engl J Med 364:501-513. doi:10.1056/NEJMoa1003825

Sabatini DM, Erdjument-Bromage H, Lui M, Tempst P, Snyder SH (1994) RAFT1: a mammalian protein that binds to FKBP12 in a rapamycin-dependent fashion and is homologous to yeast TORs. Cell 78:35-43
Scherubl H et al (2013) Clinically detected gastroenteropancreatic neuroendocrine tumors are on the rise: epidemiological changes in Germany. World J Gastroenterol 19:9012-9019 doi:10.3748/wjg.v19.i47.9012

Shah M et al (2011) Everolimus in patients with advanced pancreatic neuroendocrine tumors (pNET): impact of somatostatin analog use on progression-free survival in the RADIANT-3 trial. In: ASCO Annual Meeting Proceedings vol. 15(suppl), p 4010

Siekierka JJ, Hung SH, Poe M, Lin CS, Sigal NH (1989) A cytosolic binding protein for the immunosuppressant FK506 has peptidylprolyl isomerase activity but is distinct from cyclophilin. Nature 341:755-757. doi:10.1038/341755a0

Strosberg JR et al (2011) First-line chemotherapy with capecitabine and temozolomide in patients with metastatic pancreatic endocrine carcinomas. Cancer 117:268-275. doi:10.1002/cncr.25425

Strosberg JR et al (2015) Appropriateness of systemic treatments in unresectable metastatic well-differentiated pancreatic neuroendocrine tumors. World J Gastroenterol 21:2450-2459 doi:10.3748/wjg.v21.i8.2450

Strosberg $\mathbf{J}$ et al (2017) Phase 3 trial of $177 \mathrm{Lu}$-dotatate for midgut neuroendocrine tumors. New Engl J Med 376:125-135. doi:10.1056/NEJMoa1607427

Tippeswamy R, Patil S, Sateesh CT, Shashidhara HP, Prabhudesai S, Prashanth P, Haridas KM (2015) Everolimus plus octreotide long-acting repeatable in advanced neuroendocrine tumors in the routine tertiary cancer care setting: an Indian experience. Indian J Cancer 52:359-362 doi:10.4103/0019-509X.176709

Tsai HJ, Wu CC, Tsai CR, Lin SF, Chen LT, Chang JS (2013) The epidemiology of neuroendocrine tumors in Taiwan: a nation-wide cancer registry-based study. PloS One 8:e62487. doi:10.1371/ journal.pone.0062487

Valle JW, Eatock M, Clueit B, Gabriel Z, Ferdinand R, Mitchell S (2014) A systematic review of non-surgical treatments for pancreatic neuroendocrine tumours. Cancer Treat Rev 40:376-389. doi:10.1016/j.ctrv.2013.08.007

Vandamme T et al (2016) Long-term acquired everolimus resistance in pancreatic neuroendocrine tumours can be overcome with novel PI3K-AKT-mTOR inhibitors. Br J Cancer 114:650-658. doi:10.1038/bjc.2016.25

Vignot S, Faivre S, Aguirre D, Raymond E (2005) mTOR-targeted therapy of cancer with rapamycin derivatives. Ann Oncol 16:525-537. doi:10.1093/annonc/mdi113

von Wichert $\mathrm{G}$ et al (2000) Insulin-like growth factor-I is an autocrine regulator of chromogranin A secretion and growth in human neuroendocrine tumor cells. Cancer Res 60:4573-4581

Wullschleger S, Loewith R, Hall MN (2006) TOR signaling in growth and metabolism. Cell 124:471-484. doi:10.1016/j. cell.2006.01.016

Yao JC et al (2008a) One hundred years after "carcinoid": epidemiology of and prognostic factors for neuroendocrine tumors in 35,825 cases in the United States. J Clin Oncol 26:3063-3072. doi:10.1200/JCO.2007.15.4377

Yao JC et al (2008b) Efficacy of RAD001 (everolimus) and octreotide LAR in advanced low- to intermediate-grade neuroendocrine tumors: results of a phase II study. J Clin Oncol 26:4311-4318. doi:10.1200/JCO.2008.16.7858

Yao JC et al (2010) Daily oral everolimus activity in patients with metastatic pancreatic neuroendocrine tumors after failure of cytotoxic chemotherapy: a phase II trial. J Clin Oncol 28:69-76. doi:10.1200/JCO.2009.24.2669

Yao JC et al (2011) Everolimus for advanced pancreatic neuroendocrine tumors. New Engl J Med 364:514-523. doi:10.1056/ NEJMoa1009290

Yao JC et al (2016) Everolimus for the treatment of advanced, non-functional neuroendocrine tumours of the lung or 
gastrointestinal tract (RADIANT-4): a randomised, placebocontrolled, phase 3 study. Lancet 387:968-977. doi:10.1016/ S0140-6736(15)00817-X

Yates CJ, Newey PJ, Thakker RV (2015) Challenges and controversies in management of pancreatic neuroendocrine tumours in patients with MEN1. Lancet Diabetes Endocrinol 3:895-905. doi:10.1016/S2213-8587(15)00043-1

Yuan F et al (2014) KRAS and DAXX/ATRX gene mutations are correlated with the clinicopathological features, advanced diseases, and poor prognosis in Chinese patients with pancreatic neuroendocrine tumors. Int J Biol Sci 10:957-965. doi:10.7150/ijbs.9773

Zatelli MC, Fanciulli G, Malandrino P, Ramundo V, Faggiano A, Colao A, Group N (2016) Predictive factors of response to mTOR inhibitors in neuroendocrine tumours. Endocr Relat Cancer 23:R173-183. doi:10.1530/ERC-15-0413
Zeng $\mathrm{Z}$ et al (2007) Rapamycin derivatives reduce mTORC2 signaling and inhibit AKT activation in AML. Blood 109:3509-3512. doi:10.1182/blood-2006-06-030833

Zhou C-F, Ji J, Yuan F, Shi M, Zhang J, Liu B-Y, Zhu Z-G (2010) mTOR activation in well differentiated pancreatic neuroendocrine tumors: a retrospective study on 34 cases. Hepatogastroenterology 58:2140-2143

Zitzmann K et al (2007) The novel mTOR inhibitor RAD001 (everolimus) induces antiproliferative effects in human pancreatic neuroendocrine tumor cells. Neuroendocrinology 85:54-60. doi:10.1159/000100057 